\title{
Implementation and use of Simulated Students for Test and Validation of new Adaptive Educational Systems: a Practical Insight
}

\author{
Fabiano Dorça
}

Published online: 13 February 2015

(C) International Artificial Intelligence in Education Society 2015

\begin{abstract}
Studies attest that learning is facilitated if teaching strategies are in accordance with students learning styles, making learning process more effective and considerably improving students performances. In this context, one major research point - and a challenge - is to efficiently discover students' learning styles. But, the test and validation of new approaches in this field requires substantial amounts of financial, human resources (tutors and students) and time. In this way, the use of simulated students for test and validation of new approaches in this field is very important. Therefore, this work depicts the implementation and use of simulation for empirical evaluation of three different strategies for automatic learning styles modelling. It was necessary to compare the efficiency of the strategies, in order to choose the best one. Therefore, it was needed a practical mechanism to evaluate and compare them, preferably without the engagement of human resources. Then, the main goal of using simulation in this work is to compare strategies' efficiency and to discover the most promising one. The research was empirical, and has led to considerable enhancements on an intelligent component for automatic modelling of learning and teaching styles, which has been tested, adjusted and improved in a reasonable time and with low cost. The best strategy was clearly found, as depicted by experiments and results presented in this paper.
\end{abstract}

Keywords Simulated students · Student modelling · Learning styles · Teaching styles $\cdot$ Adaptive educational systems

F. Dorça $(\bowtie)$

Faculty of Computer Science (FACOM) - Federal University of Uberlandia (UFU),

Campus Santa Monica - Bloco 1B - Sala 1B148,

Av. Joao Naves de Avila, 2.121 - Bairro Santa Monica - CEP 38400-902, Uberlandia/MG, Brazil

e-mail: fabianodor@ufu.br 


\section{Introduction}

There is a growing demand for the incorporation of intelligent aspects into educational systems, so that individualization abilities may be developed in these systems. According to Virvou et al. (2003), these systems are meant to provide individualized tutoring to students by adapting the teaching material to their specific needs and abilities, like learning styles.

The design of such features demands an extensive and carefull experimentation and validation, in order to guarantee that they work as expected. The design of such features typically require the construction of intelligent algorithms and relatively complex mathematical models. Therefore, knowing its efficiency and effectiveness requires systematic experimentation and detailed analysis of results, so one can get a safe idea about the validity of a particular approach. The conduction of experiments and tests involving human subjects may add great complexity to the work. It is widely known that running a single test using real students could take months.

A big challenge in this research field is to test and validate automatic student modelling approaches before effectively using them. In this way, careful experimentation are demanded. This issue is even more critical when considering that the demand for these systems is growing fast, and the number of students who rely on these systems grows rapidly (Graf et al. 2009). Therefore, testing and validating new approaches in the domain of adaptive educational systems have become a critical issue. In this context, one major research point - and a challenge - is to efficiently evaluate new approaches on content personalization in adaptive educational systems.

One critical aspect on content personalization is the efficient and effective learning styles modelling. Then, it is imperative that new approaches on learning styles modelling are tested and validated before being effectively implanted and used in an adaptive educational system, in order to guarantee the effectiveness of the teaching process. According to Goguadze et al. (2011), the quality of an adaptive educational system critically depends on the quality of its student modelling. In this context, computer simulation techniques are essential, since the implementation and test of new features in adaptive educational systems require substantial amounts of financial, human resources and time.

The use of simulation is gradually becoming essential for testing new approaches on this research field. It allows the correction of problems and the improvement of new models, because it allows immediate evaluations, comparisons, adjustments and corrections of problems since the very beginning of the development process. Simulation is a widespread and widely used technique for testing educational approaches and may bring advantages, as stated by Abdullah and Cooley (2002), Vanlehn et al. (1994), Vizcaino and Du Boulay (2002), Virvou et al. (2003), Bravo and Ortigosa (2006), Mertz (1997), and Meyn et al. (1996).

Considering this challenging scenario, this paper presents details on the use of simulation for experimenting different strategies proposed for automatic and dynamic learning styles modelling. The main intention on using simulation was to compare the strategies rapidly and identify the most promising one, without the engagement of human students, due to the lack of knowledge on the efficiency and reliability of each strategy. 
Therefore the main hypotheses to be tested in this work are:

- 1 . There is a most efficient and promising strategy among the proposed ones;

- 2. The most efficient and promising strategy could be discovered by the use of simulation, saving expensive resources as time, infra-structure, human tutors and students;

- 3. Simulation contributes to a deep analysis of a new model, method, approach or strategy.

In this work, the simulated student is a module of software implemented through an expert system that encapsulates knowledge about how learning styles affect student performance in learning. It infers the student performance according to its learning styles, considering the teaching style provided by the system during a learning session. The inference is probabilistic and takes into account some available studies on how learning styles may affect student performance, as discussed by Felder and Silverman (1988), Haider et al. (2010), Graf et al. (2008), Kinshuk and Graf (2009), Graf and Liu (2008), and Bajraktarevic et al. (2003). The simulated student has been successfully used for testing automatic approaches for learning styles modelling and teaching styles selection. This experience is fully depicted in this paper.

Next section introduces some theoretical foundation related to this work.

\section{Theoretical Foundation}

\section{Learning Styles}

Recent studies claim that learning is improved if teaching strategies are in accordance with student's learning styles (Haider et al. 2010; Graf et al. 2008; Kinshuk and Graf 2009; Graf and Liu 2008; Bajraktarevic et al. 2003). According to them, it makes learning process more effective and considerably improves student performance. Therefore, when there is a significant mismatch between teaching strategies and student's preferences, the student becomes inattentive, bored or discouraged, and performs poorly.

There are many different theories and models of learning styles with varying dimensions and variables. They focus on different aspects, cognitive processes, skills, sensory modalities, learning processes and thinking styles. The term learning style may include more than 70 different models with conflicting assumptions about learning, and with different designs. Theories on how learning styles may affect student performance assume that everyone can learn, but in different ways and levels (Bostrom 2011).

Well-known theories and models of learning styles have been proposed by Kolb and et al. (1984), (Honey and Mumford 1992), (Entwistle 1981), (Pask 1976) and (Felder and Silverman 1988). Each one of these models describes different aspects in which students prefer to learn. In this work, we consider the Felder and Silverman's definition, where learning styles are defined as the preferences in the way people receive and process information (Felder and Silverman 1988). 
Graf and Kinshuk. C. (2009) point out that Felder and Silverman's Learning Styles Model (FSLSM) is one of the most frequently used in adaptive educational systems (Brusilovsky 2001). Besides, Kuljis and Liu (2005) claim that the FSLSM is the most appropriate model for the implementation of adaptive educational systems. According to Kinshuk and Graf (2009), the FSLSM combines features from the main models, such as Kolb and et al. (1984), Pask (1976), and Myers et al. (1985).

According to Graf and Kinshuk. C. (2009) and Graf and Kinshuk (2010), the FSLSM uses the concept of dimensions, and therefore describes learning styles more thoroughly. As proposed by Felder and Silverman (1988), each learner has a dominant preference in each of the four dimensions: processing (active/reflective); perception (sensitive/intuitive); input (visual/verbal); understanding (sequential/global). Each preference tells us about how a student learns best, and the related teaching strategies for effective learning. According to FSLSM, each preference within the scope of the four dimensions described above is measured on a scale from 0 to 11 , according to the answers given by the student in the Index of Learning Styles Questionnaire (ILSQ). This characteristic makes it possible to describe the strength of the learners' preferences. Kinshuk and Graf (2009).

As described by Graf and Kinshuk. C. (2009), learning styles in the FSLSM are considered to be flexibly stable, which means that they are relatively stable but they can change over time, for example, when learners trains their weak, or balanced, preferences. Furthermore, the FSLSM is based on the concept of tendencies, which means that even a strong active learner can, sometimes, act in a reflective way (Graf and Kinshuk. C. 2009).

An important characteristic of the FSLSM for this work is that it uses scales to classify students, instead of using fixed types. In this way, the strength of each preference can be finely measured (Felder and Silverman 1988). It means that the student doesn't have a fixed preference for one or other learning style. The student have a major or minor tendency for one learning style. The strength of this tendency may vary on the time, as student evolve, or train weakness. Another important aspect of the FSLSM is that it considers learning styles as tendencies, which means that students may act differently in specific situations, in other words, in a non-deterministic way, as pointed out by Kinshuk and Graf (2009). Therefore, there is a fuzzy aspect enclosed in the learning styles modelling and in the selection of appropriate teaching strategies.

\section{Stochastic Simulation}

Research reveals that stochastic models are important tools for representation of the real world. Due to fast and inexpensive computational power, the best approach is to model a real phenomenon as faithfully as possible, and then rely on a simulation study to analyse it. Prodan and Prodan (2001) alerts about the advantages of stochastic models for representation of real world activities.

Simulation methods have great importance in a diversity of projects. Stochastic simulation is an analysis mechanism for evaluate non-deterministic models. 
This models, even called stochastic models, allow the representation of uncertainty variables and their values. Because of these uncertainty variables, every time that a non-deterministic model is evaluated its behaviour can change, even if the same parameters' values are provided.

Being stochastic implies in being or having a random variable. In this way, stochastic simulation considers the generation of random numbers in order to explore the uncertainty space of a given phenomenon such that behaviour may be mathematically quantified. Stochastic simulation is the art of generating samples of random variables in a computational environment, and use them to obtain of a certain result (Geiss 2009).

In this kind of process, random variables with uniform distribution in the interval $[0,1]$ are used in different ways. Commonly, they are used to generate discrete and continuous distributions, and to generate set of dependent variables (Prodan and Prodan 2001).

There are three levels of simulation to be considered. The first level consists of simulating random numbers, as they are the basis of any stochastic simulation study. Based on the first level, the second level of simulation applied for distributional models is built. Prodan and Prodan (2001) presents the most commonly used distributional models. The third level of simulation is devoted to applications. As an application, this work presents the simulated student module.

\section{Related Works}

Some works, such as Abdullah and Cooley (2002), Vanlehn et al. (1994), Vizcaino and Du Boulay (2002), Virvou et al. (2003), Bravo and Ortigosa (2006), and Mertz (1997) propose different simulation approaches for evaluating adaptive educational systems.

Abdullah and Cooley (2002) proposes the simulation of three different types of students: one that possesses all the relevant skills in the domain, one that holds a subset of the relevant skills, and one which possess some wrong knowledge about the domain. Then, it was verified whether the system behaved properly, considering each type of student.

Vanlehn et al. (1994) discusses about the use of machine learning techniques to build students simulators, and comments about different applications of them: testing new systems, teachers training in tutoring, and collaborative learning through the interaction of simulated students with real students.

Vizcaino and Du Boulay (2002) present a simulated student used to encourage collaborative participation of human students in the teaching-learning process, checking their knowledge and helping them to solve exercises, besides analysing messages posted in chats and interacting directly with them in this environment.

Virvou et al. (2003) present an evaluation agent that simulates students behaviour in Intelligent Tutoring Systems. The authors present an approach for evaluating elearning systems through simulation-based techniques for modelling real students 
through cognitive characteristics (level of knowledge, memorization ability and retention of knowledge) and temperamental characteristics (how the student behaves and interacts with the system).

Bravo and Ortigosa (2006) use simulation in order to create log files from the interaction of fictitious students with the system. These log files are then used by data mining systems, which verify the efficiency of an adaptive educational system in providing adaptivity.

Mertz (1997) presents a cognitive model used in the simulation of students in order to assist the evaluation of lessons developed by human tutors. That is, tutors can test the lessons developed before making them available in a course.

However, none of these studies take into account learning styles for inferring student performance and behaviour. Therefore, this work focuses in the description of a stochastic model that reacts to events in a simulated learning process. The main intention is to reproduce the performance of students during the interaction with a fictitious learning environment, in order to test the effectiveness of three different strategies for automatic learning styles modelling. The stochastic characteristic of the proposed model enables to simulate the uncertainty aspect related to the student behaviour and performance. This aspect is essential when simulating student performance for testing new approaches for automatic modelling of students.

Considering the learning process as a non-deterministic process, which is influenced by many factors, the simulated student presented in this work behaves in a way that the occurrence of inadequately adapted content may contribute to its failure, but, cannot determine it. Some of the factors that exert influence on the learning process are pointed out by Al-Dujaily and Ryu (2006), Lim et al. (2007), Zhang et al. (2010), Santos and Boticario (2008), and Mosakhani and Jamporazmey (2010). These factors are taken into account by the simulated student, which considers the non-deterministic aspects involved in student performance. The uncertainty about student actions and performance is even more critical when considering e-learning, in which the teacher doesn't have close contact with students.

This work focus on addressing these points. As a result, it was possible to find the best strategy for learning style modelling among three different strategies initially proposed. This is an innovative point of this work in relation to the others. Comparing different strategies in order to decide which is the best one, and discard unsatisfactory strategies, is essential and it must be done rapidly. This implies in the use of simulation, avoiding the use of human students. Human students should enter in process only when the quality of an approach is attested, avoiding time losing and unnecessary human effort. This is the main subject of this work and the next section depicts how this point was addressed.

\section{Modelling Learning Styles and Selecting Appropriate Teaching Styles}

The student model is essential for an adaptive educational system. It enables the system to provide adapted content to individual students. The student model considered in this work is restricted to leaning styles, and it is based on the Felder and Silverman Learning Styles Model (FSLSM) (Felder and Silverman 1988). Graf and Kinshuk. C. 
(2009) and Graf and Kinshuk (2010) discusses some important aspects and advantages of this model in the implementation of adaptive educational systems. However, this work could have been realized considering other learning styles theories.

An important characteristic of FSLSM to this work is that it uses scales instead of fixed types. In this way, the strength of each preference can be measured (Felder and Silverman 1988). FSLSM considers learning styles as tendencies (Kinshuk and Graf 2009; Graf and Kinshuk. C. 2009). Therefore, every student has a certain preference for every pole of a dimension.

These tendencies are treated in this work as probabilities, being called here as student's probabilistic learning styles $\left(L S_{p}\right) . L S_{p}$ represents the probability of preference for each learning style by a specific student. Thus, a probabilistic student model is considered in this work, in which learning styles are processed by the system as probabilities, and not as certainties.

Table 1 shows an example (where "A" is Active; "R" is Reflective; "S" is Sensitive; "I" is Intuitive; "Vi" is Visual; "Ve" is Verbal; "Seq" is Sequential and "G" is Global).

This model can be initialized through cold start, setting these probabilities to 0.50 . Or, it can be initialized using the results of Index of Learning Styles Questionnaire (ILSQ). In this case, it is necessary to transform the obtained results into percentages, which denote probabilities in our approach, as it can be seen in next section. It can be easily done by dividing by 11 the number of answers favourable to each learning style, considering that the ILSQ has 11 questions for each dimension of the FSLSM, totalling 44 questions.

\section{Selecting Teaching Styles}

Considering this probabilistic student model, there are 16 possible Learning Styles Combinations (LSC), such that $\mathrm{LSC}=(\mathrm{A}, \mathrm{S}, \mathrm{Vi}, \mathrm{Seq}), \quad(\mathrm{A}, \mathrm{S}, \mathrm{Vi}, \mathrm{G}), \quad(\mathrm{R}, \mathrm{S}, \mathrm{Vi}, \mathrm{Seq})$, $(\mathrm{R}, \mathrm{S}, \mathrm{Vi}, \mathrm{G}), \quad(\mathrm{A}, \mathrm{S}, \mathrm{Ve}, \mathrm{Seq}), \quad(\mathrm{A}, \mathrm{S}, \mathrm{Ve}, \mathrm{G}), \quad(\mathrm{R}, \mathrm{S}, \mathrm{Ve}, \mathrm{Seq}), \quad(\mathrm{R}, \mathrm{S}, \mathrm{Ve}, \mathrm{G}),(\mathrm{A}, \mathrm{I}, \mathrm{Vi}, \mathrm{Seq})$, (A,I,Vi,G), (R,I,Vi,Seq), (R,I,Vi,G), (A,I,Ve,Seq), (A,I,Ve,G), (R,I,Ve,Seq), $(\mathrm{R}, \mathrm{I}, \mathrm{Ve}, \mathrm{G})$. Therefore, a learning style combination is formed by a learning style from each dimension of FSLSM, representing different combinations of preferences.

At each learning session, the student must interact with a set of learning objects (LO) (IEEE 2010), which satisfy a specific learning style combination, stochastically selected according to the student's $L S_{p}$. A learning session is a small part of a course, designed to teach a specific part of the knowledge domain, and allowing students reaching a specific learning goal. A learning goal represents a piece of the knowledge domain that must be learned by the student during the course.

Table 1 Student's Probabilistic Learning Styles $\left(L S_{p}\right)$

\begin{tabular}{|c|c|c|c|c|c|c|c|}
\hline \multicolumn{2}{|c|}{$\begin{array}{l}L S_{p} \\
\text { Processing }\end{array}$} & \multicolumn{2}{|c|}{ Perception } & \multicolumn{2}{|l|}{ Input } & \multicolumn{2}{|c|}{ Understanding } \\
\hline (A) & $(\mathrm{R})$ & (S) & (I) & (Vi) & $(\mathrm{Ve})$ & $(\mathrm{Seq})$ & (G) \\
\hline 0.35 & 0.65 & 0.17 & 0.83 & 0.89 & 0.11 & 0.84 & 0.16 \\
\hline
\end{tabular}


The probability of selecting a specific learning style combination in a learning session is given by (1), such that: $a$ refers to a learning style in the dimension Processing; $b$ refers to a learning style in the dimension Perception; $c$ refers to a learning style in the dimension Input; and $d$ refers to a learning style in the dimension Understanding.

$$
P(a, b, c, d)=P r_{a} \times P r_{b} \times P r_{c} \times P r_{d}
$$

Therefore, a learning style combination can be selected in the beginning of a learning session according to the probability distribution shown in Table 2, considering the student model presented in Table 1 . Table 2 presents the probability of selection of each learning style combination. As it is a probability distribution, the sum of the probabilities must equal 1 .

At each learning session, a new learning style combination is selected through a simple stochastic selection method, the roulette wheel selection (Goldberg 1989). The selected learning style combination points out the teaching style that will be adopted during a learning session. The teaching style dictates the kind of material that will be presented to the student, and how it will be presented. Thus, the closer the selected learning style combination from the student preferences, the better the content adaptation.

The probability distribution (Table 2) guides the adaptive process, which is a stochastic process with discrete states. It means that previous states are irrelevant for the prediction of the following states, since the current state is known. In this case, the state space is represented by LSC, with a total of 16 states. As the learning process evolves, $L S_{p}$ is automatically updated. Therefore, the LSC's probability

Table 2 LSC's Probability Distribution

\begin{tabular}{ll}
\hline LSC & Probabilities \\
\hline P(A,S,Vi,Seq) & $0,35 \times 0,17 \times 0,89 \times 0,84=0,045$ \\
P(A,S,Vi,G) & $0,35 \times 0,17 \times 0,89 \times 0,16=0,008$ \\
P(R,S,Vi,Seq) & $0,65 \times 0,17 \times 0,89 \times 0,84=0,083$ \\
P(R,S,Vi,G) & $0,65 \times 0,17 \times 0,89 \times 0,16=0,016$ \\
P(A,S,Ve,Seq) & $0,35 \times 0,17 \times 0,11 \times 0,84=0,005$ \\
P(A,S,Ve,G) & $0,35 \times 0,17 \times 0,11 \times 0,16=0,002$ \\
P(R,S,Ve,Seq) & $0,65 \times 0,17 \times 0,11 \times 0,84=0,010$ \\
P(R,S,Ve,G) & $0,65 \times 0,17 \times 0,11 \times 0,16=0,003$ \\
P(A,I,Vi,Seq) & $0,35 \times 0,83 \times 0,89 \times 0,84=0,217$ \\
P(A,I,Vi,G) & $0,35 \times 0,83 \times 0,89 \times 0,16=0,043$ \\
P(R,I,Vi,Seq) & $0,65 \times 0,83 \times 0,89 \times 0,84=0,403$ \\
P(R,I,Vi,G) & $0,65 \times 0,83 \times 0,89 \times 0,16=0,076$ \\
P(A,I,Ve,Seq) & $0,35 \times 0,83 \times 0,11 \times 0,84=0,026$ \\
P(A,I,Ve,G) & $0,35 \times 0,83 \times 0,11 \times 0,16=0,005$ \\
P(R,I,Ve,Seq) & $0,65 \times 0,83 \times 0,11 \times 0,84=0,049$ \\
P(R,I,Ve,G) & $0,65 \times 0,83 \times 0,11 \times 0,16=0,009$ \\
Sum of probabilities of all LSC & 1.000 \\
\hline
\end{tabular}


distribution changes constantly, which causes changes on probabilities of transition between states.

\section{Evolving the Student Model}

The student model is evolved by a process based on reinforcement learning (Sutton and Barto 1998). Three strategies for updating $L S_{p}$ according to student performance were implemented and tested:

- 1. reinforce the $L S_{p}$ that appear in selected LSC when student obtain good performance.

- 2. reinforce the $L S_{p}$ that appear in selected LSC when student obtain good performance, and reinforce the $L S_{p}$ that don't appear in selected LSC when student obtain poor performance.

- 3. reinforce the $L S_{p}$ that don't appear in LSC when student obtain poor performance.

Poor performance occurs when student performance is lesser than $m$, such that $m$ is the threshold between good and bad performance.

Knowing the most efficient strategy was an important issue in this work. In this way we could concentrate in the most promising, and discard the others. This scenario really encouraged us on the use of simulation, in order to deeply analyse the behaviour of each strategy, and decide which should be chosen.

In the strategies presented above, the reinforce of $L S_{p}$ was done through adding a reinforcement $R$ to $L S_{p}$. The reinforcement $R$ to be applied in $L S_{p}$ is calculated according to the performance $(\mathrm{P})$ obtained by the student during a learning session. As the values of $L S_{p}$ represent probability distributions related to student preferences for each dimension, when a $L S_{p}$ in a dimension is reinforced, its pair in the dimension is reduced by the same value, maintaining their sum equal 1 .

For example, considering the $L S_{p}$ presented in Table 1, considering that the LSC (A,S, Vi,Seq) was selected, considering that the strategy 3 was in use, considering that the student obtained poor performance, and considering that the value of $R$ was 0.09 , then the resulting $L S_{p}$ is presented in Table 3 .

Observe that the $L S_{p}$ that don't appear in LSC were incremented by 0.09 , and automatically the $L S_{p}$ that appear in LSC were decremented by the same value, keeping consistent probability distributions. The updating of the $L S_{p}$ causes the re-computation of LSC's probability distribution, shown in Table 2. Consequently, different LSC may be selected, due to their increase of probability. The main goal of

Table 3 Student's Probabilistic Learning Styles $\left(L S_{p}\right)$

\begin{tabular}{|c|c|c|c|c|c|c|c|}
\hline \multicolumn{2}{|c|}{$\begin{array}{l}L S_{p} \\
\text { Processing }\end{array}$} & \multicolumn{2}{|c|}{ Perception } & \multicolumn{2}{|l|}{ Input } & \multicolumn{2}{|c|}{ Understanding } \\
\hline (A) & $(\mathrm{R})$ & (S) & (I) & (Vi) & $(\mathrm{Ve})$ & $(\mathrm{Seq})$ & (G) \\
\hline 0.26 & 0.74 & 0.08 & 0.92 & 0.80 & 0.20 & 0.75 & 0.25 \\
\hline
\end{tabular}


this process is to converge $L S_{p}$ to the student actual preferences, making the adaptive process as effective as possible.

$R$ is inversely related to performance, since, probably, the lower the performance, the greater the difficulty of learning, which can probably be caused by strong inconsistency in $L S_{p}$, which should be eliminated as soon as possible, requiring greater reinforcement. It was important to limit the value of $R$ in order to avoid abrupt changes in $L S_{p}$, which could lead to inconsistency in some dimensions. In this way, gradual changes in the values of $L S_{p}$ were essential.

In order to test and compare the three strategies presented above, a simulated student module has been implemented and used. The simulated student interacts with a fictitious course, and its performance at each learning session is inferred by a stochastic model, taking into account how the course attends to its preferences. The simulated student is depicted in next section.

\section{Simulating Students Based on Learning Styles}

Before implementing this student modeling approach in an adaptive educational system and using it with real students, it was really important to test it using a simulated process. Testing it through a simulated process was vital to verify how efficiently the presented strategies could:

- model student's LS

- select the most appropriate teaching style

- diminish learning difficulties, or learning problems

- improve the overall student's performance

Testing it on a simulated way enabled to quickly find the best strategy, and make modifications and adjustments in its mathematical model and parameters, in order to improve its efficiency. Many variables and parameters were involved, and adjusting them was essential in order to guarantee the efficiency of the proposed model. As we know, if estimations about the student's LS are incorrect, the adaptive process are unlikely to be effective, because inadequate teaching styles could be selected.

As showed in the previous section, considering the presented student modelling approach, the only necessary variable to test it is the student performance, which is used to calculate the reinforcement $R$. Therefore, the reasonable estimation of the performance during the test is the main goal of the simulated student module depicted in this section. Thus, the goal is to observe whether the proposed approach can effectively improve the performance of students in the course. The simulated student allowed to compare the approach presented in this paper with other approaches, and continuously evolve it.

The simulated student is implemented as an expert system which encapsulates knowledge about students behaviour considering their learning styles and the teaching styles provided for them. The knowledge necessary was mainly obtained from Haider et al. (2010), Graf et al. (2008), Kinshuk and Graf (2009), Graf and Kinshuk. C. (2009), Graf and Liu (2008), Coffield et al. (2009), Alfonseca et al. (2006), Graf 
and Lin (2007), Graf and Kinshuk (2007), Bajraktarevic et al. (2003), Sangineto et al. (2008), Terry et al. (1995), Friedel and Rudd (2006), and Vasilyeva et al. (2006).

The main characteristic of the simulated student is that when the student's LS are met by the selected teaching style, learning becomes easier, and the probability of success is increased. It means that the student is benefited when his/her learning styles are supported by the learning environment. As pointed out by Graf et al. (2008), strong preferences produce stronger negative effects on the student's performance when these preferences are unmet by the teaching style, and this fact is modelled by the simulated student. The impact of LS strengths on students' performances is analysed by Felder and Silverman (1988), Haider et al. (2010), Graf et al. (2008), and Kinshuk and Graf (2009).

Studies have shown that learners with strong preferences on a specific LS have more difficulties to learn than learners with mild preferences. According to Kinshuk and Graf (2009), this finding shows that learners with strong LS preferences can be especially benefited from adaptivity. According to Kinshuk and Graf (2009), in mismatched courses these students scored significantly lower on the final exam than students who did not have preferences as pronounced. This result confirms Felder's argument about the importance of adaptivity with respect to LS, especially for students who have strong preferences (Felder and Silverman 1988). In the same way, unmet moderate preferences tend to cause greater negative impact than unmet week (or balanced) preferences, since in this type of preference the student tends to have dexterity in both LS in a dimension, as stated by Kinshuk and Graf (2009).

The simulated student experiences an increase of difficulty when its LS are unmet by the currently selected teaching style. In this way, the simulated student infers the degree of difficulty to be faced during a learning session in the simulated learning process. Increasing the probability of failure also increases the level of difficulty. Therefore, considering the learning process as a non-deterministic process, which is influenced by many factors besides LS, the simulated student behaves in a way that the occurrence of inadequately adapted content may contribute to its failure, but, cannot determine it.

Some of the factors that exert influence on the learning process are pointed out by Al-Dujaily and Ryu (2006), Lim et al. (2007), Zhang et al. (2010), Santos and Boticario (2008), and Mosakhani and Jamporazmey (2010). These factors are taken into account by the simulated student, which considers the nondeterministic aspects involved in students' performances evaluations and learning processes.

Before running the simulated student, the student's real LS $\left(L S_{r}\right)$ and the strength of each preference (strong, moderate, weak or balanced) are set. These strengths exert influence on the outcome student's performances, as stated before. In this context, the simulated student considers the amount of unmet LS $\left(L S_{u}\right)$ between its $L S_{r}$ and the currently selected LSC.

As FSLSM has four dimensions, it is possible to occur between 0 and 4 unmet LS. For example, if $L S_{r}=(\mathrm{A}, \mathrm{S}, \mathrm{Vi}, \mathrm{Seq})$ and $\mathrm{LSC}=(\mathrm{R}, \mathrm{I}, \mathrm{Ve}, \mathrm{G})$, then there are 4 unmet LS $\left(L S_{u}=4\right)$. By the other way, if $L S_{r}=(\mathrm{A}, \mathrm{S}, \mathrm{Vi}, \mathrm{Seq})$ and LSC $=(\mathrm{A}, \mathrm{S}, \mathrm{Vi}, \mathrm{Seq})$, then there are 0 unmet $\mathrm{LS}\left(L S_{u}=0\right)$. In the latter case, the simulated student will face less difficulty in the learning session, because its 
preferences are attended by the system. Thus, its performance will probably be better.

A performance $(\mathrm{P})$ is given by (2).

$$
P=M-(D \times K \times \alpha)
$$

such that:

- $\quad M$ is the maximum performance. In this work, $M=100$.

- $D$ is a difficulty factor in the range [0,5], and it is given by (3).

- $\quad K$ is a constant value. If $K=20$ and $M=100$, then $\mathrm{P}$ is in the range $[0,100]$.

- $\alpha$ is a random number in the range $[0,1]$.

It is important to mention that $\alpha$ represents a random difficulty factor, which is applied because not only LS but also many factors exert some influence on students' performances, making it harder to infer students' LS based only on fixed behavioural pattern rules (Haider et al. 2010; Graf et al. 2008; Kinshuk and Graf 2009; Alfonseca et al. 2006; Graf et al. 2009; Messick 1976).

$$
D=L S_{u}+\beta+\gamma
$$

such that:

- $L S_{u}$ is the amount of unmet LS between $L S_{r}$ and the currently selected LSC, being in the range $[0,4]$.

- $\quad \beta$ - If there is at least one strong unmet preference, then $\beta$ is a random number in the range $[0,1]$. Else, $\beta$ is equal to 0 .

- $\quad \gamma$ - If there is at least one moderate unmet preference, then $\gamma$ is a random number in the range $[0,1-\beta]$. Else, $\gamma$ is equal to 0 .

- If $D=0$ then $D=$ random $\Re$ in $[0,1]$.

$\beta$ and $\gamma$ allows the simulated student to behave in a way such that strong preferences may exert stronger impact in its performances than moderate preferences. And, moderate preferences may exert stronger impact than week preferences, as stated by Felder and Silverman (1988), Haider et al. (2010), Graf et al. (2008), and Kinshuk and Graf (2009). Furthermore, $D$ is increased as $L S_{u}$ is increased, which means that unmet preferences may increase the difficulty of the learning process, and probably decrease student's performance. If $D$ is valued as 0 (when $L S_{u}$ is equal to 0 ) - student's LS fully met by the currently selected LSC $-D$ is set to a random number in the range $[0,1]$, representing an unknown and non-deterministic probability of difficulty, which means that even if student's preferences are fully met by the selected teaching style, the student may show some degree of difficulty due to other factors, which are unknown to us, and non-deterministic. Therefore, the occurrence of inadequately adapted content may contribute to students' failure, but, cannot determine it. In this way, the simulated student is able to infer its performances stochastically, considering the uncertainty aspects related to it.

As the simulated student presented here does not consider features of a specific educational system, it can be easily adapted and reused for testing other student modelling approaches based on LS. The simulated student module presented in this paper 
is uncoupled from any educational system, being independent from any specific behaviour of students in a specific system.

The simulated student receives the selected LSC on each learning session, and returns the performance obtained by the student on the learning session. The learning styles active, sensitive, visual and sequential are internally represented by 0 . And the learning styles reflective, intuitive, verbal and global are represented by 1 . Therefore, the $\mathrm{LSCs}=\{(\mathrm{A}, \mathrm{S}, \mathrm{Vi}, \mathrm{Seq}),(\mathrm{A}, \mathrm{S}, \mathrm{Vi}, \mathrm{G}),(\mathrm{R}, \mathrm{S}, \mathrm{Vi}, \mathrm{Seq}),(\mathrm{R}, \mathrm{S}, \mathrm{Vi}, \mathrm{G})$, $(\mathrm{A}, \mathrm{S}, \mathrm{Ve}, \mathrm{Seq}), \quad(\mathrm{A}, \mathrm{S}, \mathrm{Ve}, \mathrm{G}), \quad(\mathrm{R}, \mathrm{S}, \mathrm{Ve}, \mathrm{Seq}), \quad(\mathrm{R}, \mathrm{S}, \mathrm{Ve}, \mathrm{G}),(\mathrm{A}, \mathrm{I}, \mathrm{Vi}, \mathrm{Seq}), \quad(\mathrm{A}, \mathrm{I}, \mathrm{Vi}, \mathrm{G})$, (R,I,Vi,Seq), (R,I,Vi,G), (A,I,Ve,Seq), (A,I,Ve,G), (R,I,Ve,Seq), (R,I,Ve,G)\} are internally represented by $\mathrm{LSCs}=\{(0,0,0,0),(0,0,0,1),(1,0,0,0),(1,0,0,1),(0,1,0,0)$, $(0,1,0,1),(1,1,0,0),(1,1,0,1),(0,0,1,0),(0,0,1,1),(1,0,1,0),(1,0,1,1),(0,1,1,0)$, $(0,1,1,1),(1,1,1,0),(1,1,1,1)\}$. In this way, we use a binary representation of LSCs in order to simplify the implementation.

Figure 1 shows the input and output values related to the execution of the simulated student.

As it can be seen, the simulated student is a module, which receives $L S_{r}$ and LSC, and calculates performance according to the stochastic model presented.

According to Wojtusiak et al. (2012), modelling complex systems often requires using simulation techniques that approximate real-world systems behaviour. Stochastic simulation is frequently used to model systems whose operation cannot be captured directly by deterministic rules, and thus need to be approximated probabilistically, as it occurred in this work. Important considerations on stochastic modelling and simulation are done by Geiss (2009). Next section presents experiments and results.

\section{Methods}

An application was built for tests and experiments using the simulated student, allowing the execution of the simulated learning processes. The application's user interface is shown in Fig. 2.

The application allows the adjustment of important parameters, such as the learning rate, the performance threshold $m$, the reinforcement limit $R_{\text {max }}$, and the number of concepts to be learned by the student (learning goals). The learning rate is in the range $[0,1]$ and it indicates how fast the system learns with the reinforcements (the strength of reinforcements applied on $L S_{p}$ ). The learning rate is a common feature in reinforcement learning process. $R_{\max }$ limits the value of the reinforcement with the intention of preventing too large reinforcements when student performance is very

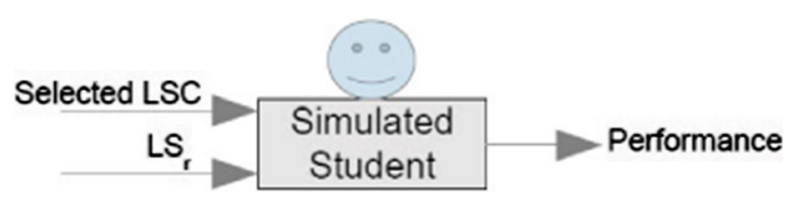

Fig. 1 Input and output values related to the execution of the simulated student 


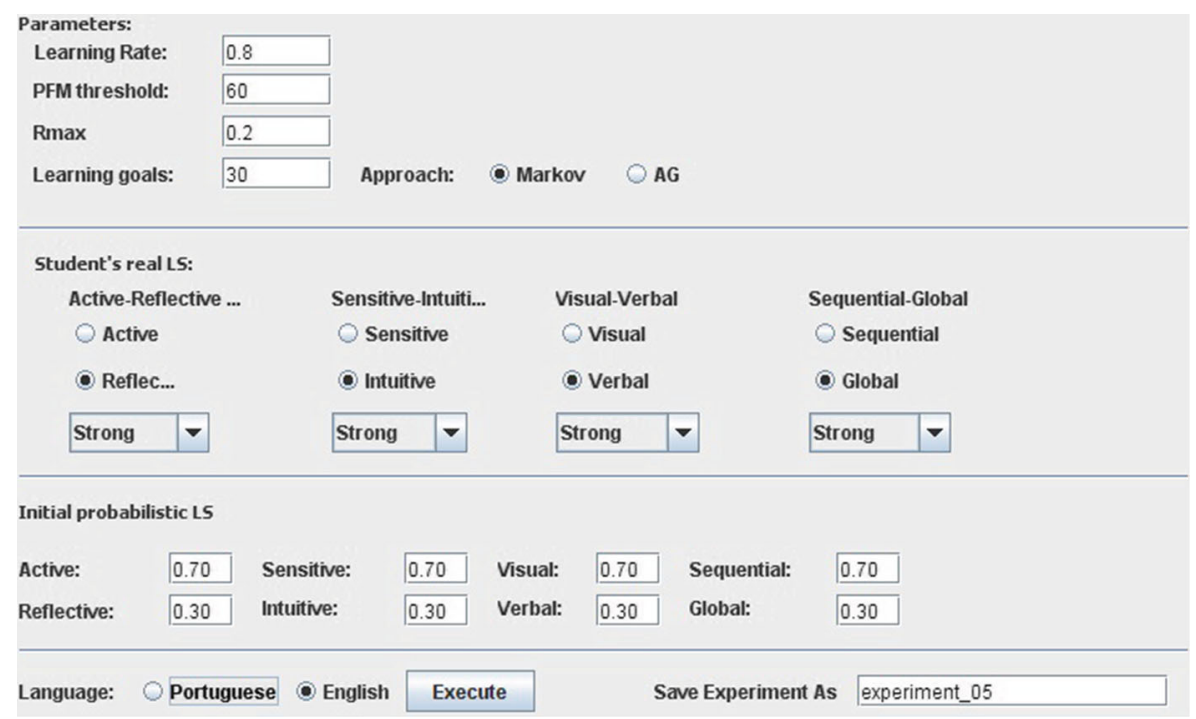

Fig. 2 Application built for tests and experiments using the simulated student

low (reinforcement and performance are inversely proportional). The main intention here is to allow a gradual modelling process, which is important to guarantee efficient convergence of $L S_{p}$ towards student's real learning styles. The application allows to set the student's real learning styles and the strength of each preference (strong, balanced or moderate).

For the experiments presented in this section, we considered that good performances occurs when $P \geq 60$, and poor performances (learning problem detected) occurs when $P<60$, which means that $m=60$ (such that $m$ is the threshold between good and bad performance). In Fig. 2, $m$ is represented by the text field labelled as "PFMthreshold". The $m$ may be configured with different values. For example, if $m=70$ it is possible to observe the occurrence of more learning problems (the student needs to obtain higher performances), and consequently, more updates will be done in $L S_{p}$. If $m=50$ it is possible to observe the occurrence of less learning problems (the student needs to obtain lower performances), and consequently, less updates will be done in $L S_{p}$. If $m$ is very low (e.g. less than 50), few learning problems are detected, and consequently, few updates in $L S_{p}$ are applied, which retards the student modelling process. On the other hand, if $m$ is very high (e.g. greater than 70 ), many learning problems are detected, and consequently, many updates in $L S_{p}$ are applied, which can disturb the convergence of $L S_{p}$ to the real learning styles.

The learning rate, which sets how fast the algorithm learns about the student's LS, was set to 0.8 , and the limit value for the reinforcement to be applied in $L S_{p}, R_{\max }$ was set to 0.2 . The constant $K$ was set to $K=20$. In this way, as $0 \leq L S_{u} \leq 5$, we have, then, $0 \leq P \leq 100$.

We considered a set of 30 concepts to be learned by the student. In each concept, the student should progress through 6 levels. Therefore, the simulated learning 
process in these experiments must have at least 180 learning sessions, or iterations (30 concepts $\times 6$ levels $=180$ iterations/learning sessions). It is, on purpose, a long course, which allows visually observing the behaviour of $L S_{p}$ during a long period. This analysis is important, and allowed to observe that $L S_{p}$ maintain consistence along the course after becoming consistent. If $P<m$ is obtained, the student must repeat the learning session, which causes an increasing on the total number of iterations necessary to finish the course. Therefore, the easier the learning process, fewer iterations are needed to finish the course.

The concepts are fictitious. There is no specific domain involved in the simulation. It is free of domain. This is a great advantage of using simulation in this context. There is no need to have real learning objects and content. They are simulated too.

The Algorithm 1 summarizes the experiments. Each experiment runs until the student learns all concepts. At the beginning of the process, $L S_{p}$ is initialized, and $L S_{r}$ is set. At each learning session, the simulated student receives a selected LSC, and returns the performance obtained on the learning session.

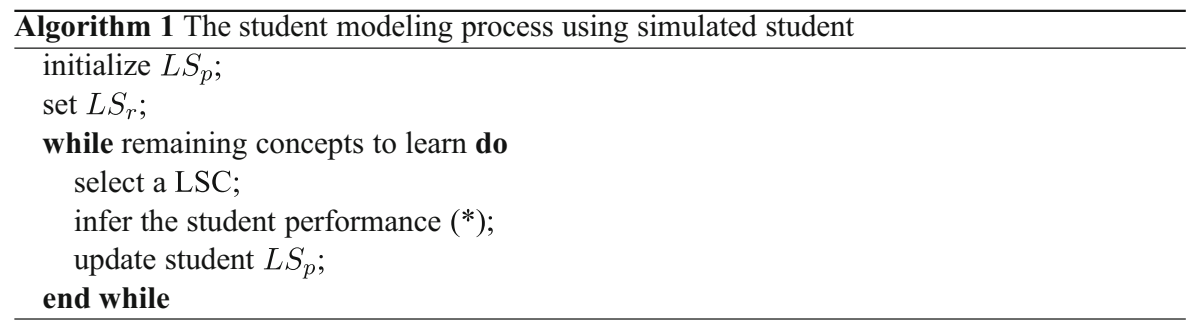

As it can be seen, the basic idea is to iterate while the student should learn something. The performance obtained by the student is used to update $L S_{p}$, according to one of the three strategies presented. The line marked with an asterisk shows where the simulated student acts.

Three aspects were carefully observed and analyzed in order to compare the strategies:

- consistency: Does $L S_{p}$ effectively converge to $L S_{r}$ during the learning process?

- efficiency: Does $L S_{p}$ converge to $L S_{r}$ in a reasonable time, i.e., $L S_{p}$ becomes consistent at the beginning of the learning process?

- maintenance: Does LSp maintain consistency after becoming consistent?

Following it is presented experiments that analyse the strategies for updating $L S_{p}$ considering these aspects. The experiments were divided in two phases. Initially, in the first phase, the goal was to identify the more promising approach in terms of consistency. In this phase, the hypotheses 1 and 2, presented in introduction, are proved. Then, in the later phase, efficiency and maintenance of the best found strategy is analysed, showing that simulation in this context may contribute to a rich analysis of a new model, method, approach or strategy. In this phase the hypothesis 3 is proved. 


\section{Experiments and Results}

Table 4 presents the initial $L S_{p}$ for this experiment. The $L S_{r}$ considered in this experiments was $\{$ Reflective (strong), Intuitive (strong), Verbal (moderate), Global (balanced) \}. Therefore, initial $L S_{p}$ is inconsistent in its four dimensions. An inconsistent $L S_{p}$ means that its value does not corresponds to the student's real preference. For example, in Table 4, the value of the Active $L S_{p}$ is 0.70 , which suggests that the student probably is an active student. But, the student's real preferences points that the student is a strong reflective. Therefore we have an inconsistency between the student model ( $L S_{p}$ values) and the student's real preferences. The main goal of the student modeling process, previously presented, is to converge $L S_{p}$ to consistent values during the student modeling process. This initial test is a extreme test, in which we have an initial student model totally inconsistent, in its four dimensions.

The main goal was to identify which strategy would obtain consistent $L S_{p}$ more frequently. Therefore, in this test the strategies are analysed in terms of their consistency aspect. In this experiment, the hypotheses 1 and 2 presented in introduction are tested, and proved.

A comparison of the three strategies is following presented. The three strategies were previously described in this paper.

\section{Experimenting Strategy 01}

Table 5 shows the number of iterations, the amount of learning problems occurred, and resulting $L S_{p}$ values at the end of the learning process, in 10 executions of this experiment. The $L S_{p}$ values marked with an asterisk were not corrected during the learning process, remaining inconsistent at the end of the process.

As it can be seen, the inconsistency level of resulting $L S_{p}$ is very high. It shows, then, that this strategy is ineffective for LS modelling.

\section{Experimenting Strategy 02}

Table 6 shows the number of iterations, the amount of learning problems occurred, and resulting $L S_{p}$ values at the end of the learning process in 10 executions of this experiment. We can notice a better level of consistency in these results, when compared to results obtained through strategy 01 . Resulting $L S_{p}$ values obtained at execution N.3 and N.5 were fully consistent. In the other executions, one inconsistency remained in resulting $L S_{p}$ Table 6 .

Table 4 Initial $L S_{p}$

\begin{tabular}{|c|c|c|c|c|c|c|c|}
\hline \multicolumn{8}{|l|}{$L S_{p}$} \\
\hline (A) & $(\mathrm{R})$ & (S) & (I) & (Vi) & $(\mathrm{Ve})$ & $(\mathrm{Seq})$ & (G) \\
\hline 0.70 & 0.30 & 0.70 & 0.30 & 0.70 & 0.30 & 0.70 & 0.30 \\
\hline
\end{tabular}


Table 5 Results obtained using strategy 01

\begin{tabular}{llll}
\hline N. & Iterations & Learning problems & Resulting $L S_{p}$ \\
\hline 1 & 390 & 210 & $\left\{(0,9 ; 0,1)^{*} ;(0,9 ; 0,1)^{*} ;(0,9 ; 0,1)^{*} ;(0,9 ; 0,1)^{*}\right\}$ \\
2 & 298 & 118 & $\left\{(0,9 ; 0,1)^{*} ;(0,1 ; 0,9) ;(0,9 ; 0,1)^{*} ;(0,9 ; 0,1)^{*}\right\}$ \\
3 & 299 & 119 & $\left\{(0,9 ; 0,1)^{*} ;(0,9 ; 0,1)^{*} ;(0,1 ; 0,9) ;(0,9 ; 0,1)^{*}\right\}$ \\
4 & 322 & 142 & $\left\{(0,1 ; 0,9) ;(0,9 ; 0,1)^{*} ;(0,9 ; 0,1)^{*} ;(0,9 ; 0,1)^{*}\right\}$ \\
5 & 317 & 137 & $\left\{(0,9 ; 0,1)^{*} ;(0,9 ; 0,1)^{*} ;(0,9 ; 0,1)^{*} ;(0,1 ; 0,9)\right\}$ \\
6 & 381 & 201 & $\left\{(0,9 ; 0,1)^{*} ;(0,9 ; 0,1)^{*} ;(0,9 ; 0,1)^{*} ;(0,9 ; 0,1)^{*}\right\}$ \\
7 & 407 & 227 & $\left\{(0,9 ; 0,1)^{*} ;(0,9 ; 0,1)^{*} ;(0,9 ; 0,1)^{*} ;(0,9 ; 0,1)^{*}\right\}$ \\
8 & 404 & 224 & $\left\{(0,9 ; 0,1)^{*} ;(0,9 ; 0,1)^{*} ;(0,9 ; 0,1)^{*} ;(0,9 ; 0,1)^{*}\right\}$ \\
9 & 429 & 249 & $\left\{(0,9 ; 0,1)^{*} ;(0,9 ; 0,1)^{*} ;(0,9 ; 0,1)^{*} ;(0,9 ; 0,1)^{*}\right\}$ \\
10 & 374 & 194 & $\left\{(0,9 ; 0,1)^{*} ;(0,9 ; 0,1)^{*} ;(0,9 ; 0,1)^{*} ;(0,9 ; 0,1)^{*}\right\}$ \\
Average & 377,5 & 197,5 & - \\
\hline
\end{tabular}

There was a significant reduction in the number of learning problems, consequently reducing the number of iterations of the learning process. Therefore, there was a significant efficiency gain in relation to strategy 01.

\section{Experimenting Strategy 03}

Table 7 shows the number of iterations, the amount of learning problems occurred, and resulting $L S_{p}$ values at the end of the learning process in 10 executions of this experiment. We can notice a better level of consistency in these results, when comparing them with results obtained through strategy 02 (Table 6). It shows a reduction in the number of learning problems, consequently reducing the number of iterations

Table 6 Results obtained using strategy 02

\begin{tabular}{llll}
\hline N. & Iterations & Learning problems & Resulting $L S_{p}$ \\
\hline 1 & 204 & 24 & $\left\{(0,1 ; 0,9) ;(0,9 ; 0,1)^{*} ;(0,1 ; 0,9) ;(0,1 ; 0,9)\right\}$ \\
2 & 206 & 26 & $\left\{(0,1 ; 0,9) ;(0,9 ; 0,1)^{*} ;(0,1 ; 0,9) ;(0,1 ; 0,9)\right\}$ \\
3 & 189 & 9 & $\{(0,1 ; 0,9) ;(0,1 ; 0,9) ;(0,1 ; 0,9) ;(0,1 ; 0,9)\}$ \\
4 & 215 & 35 & $\left\{(0,1 ; 0,9) ;(0,9 ; 0,1)^{*} ;(0,1 ; 0,9) ;(0,1 ; 0,9)\right\}$ \\
5 & 191 & 11 & $\{(0,1 ; 0,9) ;(0,1 ; 0,9) ;(0,1 ; 0,9) ;(0,1 ; 0,9)\}$ \\
6 & 211 & 31 & $\left\{(0,9 ; 0,1)^{*} ;(0,1 ; 0,9) ;(0,1 ; 0,9) ;(0,1 ; 0,9)\right\}$ \\
7 & 206 & 26 & $\left\{(0,1 ; 0,9) ;(0,1 ; 0,9) ;(0,9 ; 0,1)^{*} ;(0,1 ; 0,9)\right\}$ \\
8 & 210 & 30 & $\left\{(0,9 ; 0,1)^{*} ;(0,1 ; 0,9) ;(0,1 ; 0,9) ;(0,1 ; 0,9)\right\}$ \\
9 & 207 & 27 & $\left\{(0,1 ; 0,9) ;(0,1 ; 0,9) ;(0,8 ; 0,2)^{*} ;(0,1 ; 0,9)\right\}$ \\
10 & 205 & 25 & $\{(0,1 ; 0,9) ;(0,1 ; 0,9) ;(0,1 ; 0,9) ;(0,9 ; 0,1) *\}$ \\
Average & 206 & 26 & - \\
\hline
\end{tabular}


Table 7 Results obtained using strategy 03

\begin{tabular}{llll}
\hline N. & Iterations & Learning problems & Resulting $L S_{p}$ \\
\hline 1 & 208 & 28 & $\{(0,2 ; 0,8) ;(0,1 ; 0,9) ;(0,1 ; 0,9) ;(0,3 ; 0,7)\}$ \\
2 & 204 & 24 & $\{(0,2 ; 0,8) ;(0,1 ; 0,9) ;(0,2 ; 0,8) ;(0,3 ; 0,7)\}$ \\
3 & 213 & 33 & $\{(0,2 ; 0,8) ;(0,2 ; 0,8) ;(0,2 ; 0,8) ;(0,1 ; 0,9)\}$ \\
4 & 206 & 26 & $\{(0,1 ; 0,9) ;(0,2 ; 0,8) ;(0,2 ; 0,8) ;(0,2 ; 0,8)\}$ \\
5 & 201 & 21 & $\{(0,2 ; 0,8) ;(0,2 ; 0,8) ;(0,2 ; 0,8) ;(0,2 ; 0,8)\}$ \\
6 & 204 & 24 & $\{(0,2 ; 0,8) ;(0,2 ; 0,8) ;(0,2 ; 0,8) ;(0,1 ; 0,9)\}$ \\
7 & 210 & 30 & $\{(0,1 ; 0,9) ;(0,2 ; 0,8) ;(0,2 ; 0,8) ;(0,3 ; 0,7)\}$ \\
8 & 196 & 16 & $\{(0,1 ; 0,9) ;(0,2 ; 0,8) ;(0,2 ; 0,8) ;(0,2 ; 0,8)\}$ \\
9 & 200 & 20 & $\{(0,2 ; 0,8) ;(0,1 ; 0,9) ;(0,1 ; 0,9) ;(0,2 ; 0,8)\}$ \\
10 & 198 & 18 & $\{(0,2 ; 0,8) ;(0,3 ; 0,7) ;(0,1 ; 0,9) ;(0,3 ; 0,7)\}$ \\
Average & 204 & 24 & - \\
\hline
\end{tabular}

in the learning process. Therefore, we can conclude that using this strategy results in an efficiency gain when comparing it with strategies 01 and 02 .

It is possible to clearly notice that this strategy is much more efficient and effective than the others. With this result, the hypotheses presented in introduction were proved. Therefore, this is the chosen strategy to explore. Following it is presented new tests on this strategy. The main goal is to show that hypothesis 3 is true by analysing aspects of efficiency and maintenance described in the methods section.

\section{Deepening Tests with the Chosen Strategy}

As it was showed, strategy 03 is the best in terms of consistency, obtaining more consistent final results than strategies 01 and 02 . In this section, the main goal is to analyse aspects of efficiency and maintenance.

In this strategy, if poor performance is detected during a learning session, each learning style that appear in the selected LSC is decremented in $L S_{p}$, considering the existence of probable inconsistencies in the $L S_{p}$, which probably caused the selection of a possibly inadequate teaching style. Each learning style that doesn't appear in the selected LSC is incremented (reinforced) in $L S_{p}$, making it stronger, considering that the poor performance may be occurred because this learning style was not present in the selected LSC. Therefore, it has caused the selection of a possibly inadequate teaching style. In this way, the system learns how the student learns best.

Table 8 Initial $L S_{p}$ initialized with cold start

\begin{tabular}{llllllll}
\hline $\begin{array}{llllll}L S_{p} \\
\text { Processing }\end{array}$ & \multicolumn{2}{c}{} & \multicolumn{2}{c}{ Input } & & \multicolumn{2}{c}{ Understanding } \\
\hline$(\mathrm{A})$ & $(\mathrm{R})$ & $(\mathrm{S})$ & $(\mathrm{I})$ & $(\mathrm{Vi})$ & $(\mathrm{Ve})$ & $(\mathrm{Seq})$ & $(\mathrm{G})$ \\
0.50 & 0.50 & 0.50 & 0.50 & 0.50 & 0.50 & 0.50 & 0.50 \\
\hline
\end{tabular}


Table 9 Results obtained - all executions produced consistent results

\begin{tabular}{llll}
\hline N. & Iterations & LP & Resulting $L S_{p}$ \\
\hline 1 & 197 & 17 & $\{(0.2 ; 0.8) ;(0.2 ; 0.8) ;(0.1 ; 0.9) ;(0.2 ; 0.8)\}$ \\
2 & 192 & 12 & $\{(0.2 ; 0.8) ;(0.2 ; 0.8) ;(0.2 ; 0.8) ;(0.2 ; 0.8)\}$ \\
3 & 199 & 19 & $\{(0.1 ; 0.9) ;(0.3 ; 0.7) ;(0.2 ; 0.8) ;(0.2 ; 0.8)\}$ \\
4 & 198 & 18 & $\{(0.2 ; 0.8) ;(0.2 ; 0.8) ;(0.2 ; 0.8) ;(0.1 ; 0.9)\}$ \\
5 & 192 & 12 & $\{(0.2 ; 0.8) ;(0.2 ; 0.8) ;(0.2 ; 0.8) ;(0.2 ; 0.8)\}$ \\
6 & 194 & 14 & $\{(0.2 ; 0.8) ;(0.1 ; 0.9) ;(0.1 ; 0.9) ;(0.2 ; 0.8)\}$ \\
7 & 193 & 13 & $\{(0.3 ; 0.7) ;(0.2 ; 0.8) ;(0.2 ; 0.8) ;(0.1 ; 0.9)\}$ \\
8 & 194 & 14 & $\{(0.2 ; 0.8) ;(0.2 ; 0.8) ;(0.2 ; 0.8) ;(0.3 ; 0.7)\}$ \\
9 & 194 & 14 & $\{(0.4 ; 0.6) ;(0.1 ; 0.9) ;(0.2 ; 0.8) ;(0.2 ; 0.8)\}$ \\
10 & 193 & 13 & $\{(0.3 ; 0.7) ;(0.1 ; 0.9) ;(0.2 ; 0.8) ;(0.3 ; 0.7)\}$ \\
Average & 194.6 & 14.6 & - \\
\hline
\end{tabular}

In the following analysis, we present graphically details on how $L S_{p}$ is updated during the learning process. In each graph, the axis $x$ shows the number of iterations of the learning process, and the axis $y$ shows the values of $L S_{p}$ (multiplied by 100) throughout the learning process in each dimension of the FSLSM. The main goal was to observe how $L S_{p}$ is gradually updated throughout the learning process. Hence, it was possible to clearly visualize the student modelling process.
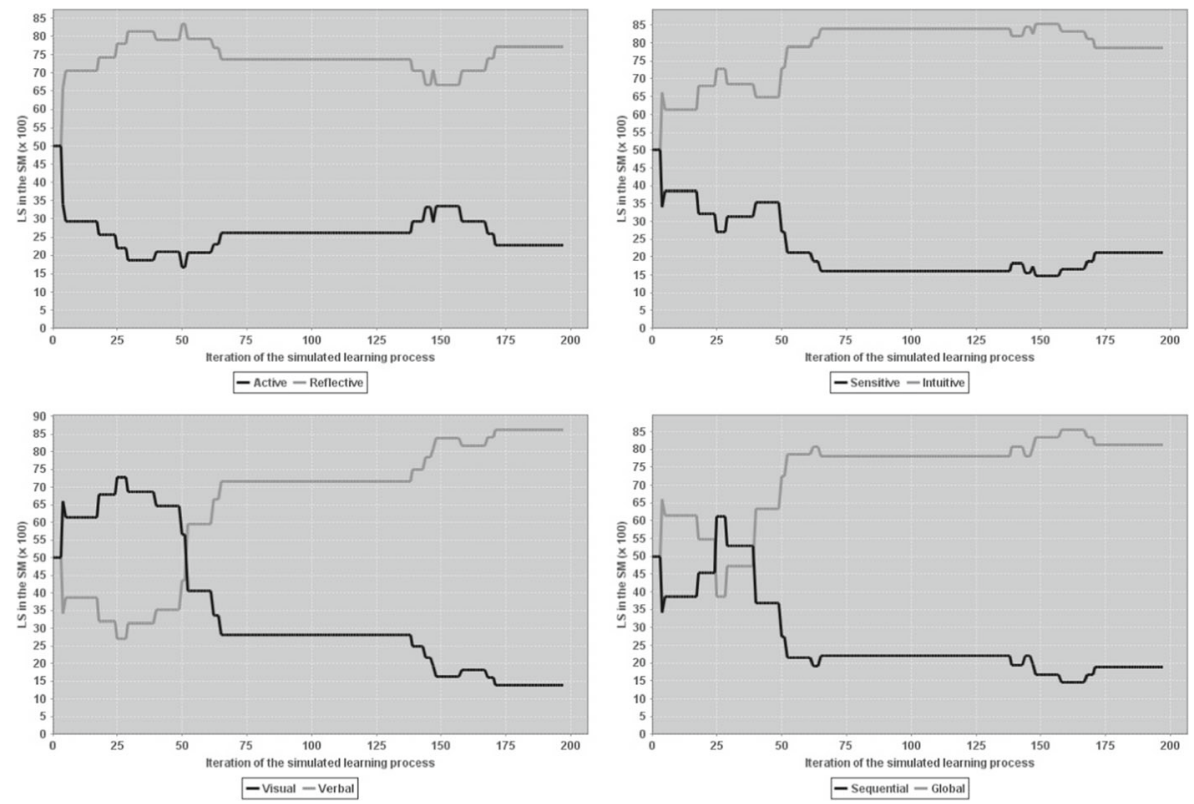

Fig. 3 Updating $L S_{p}$ throughout the simulated learning process - satisfying consistency and efficiency aspects 


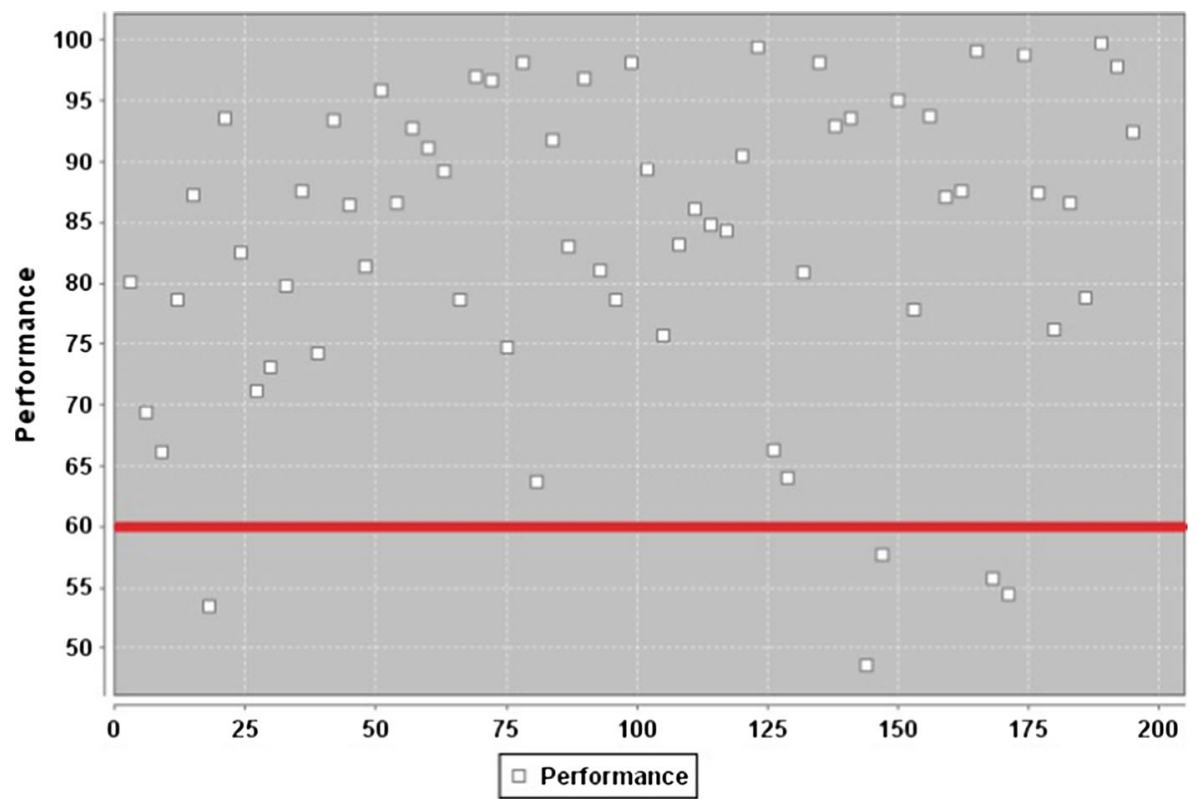

Fig. 4 Simulated Student's performances - learning problems were reduced

Moreover, for each experiment we present a graph showing the performance inferred by the simulated student at steps of 5 iterations. It is also presented the average performance obtained by the simulated student, and the average learning problems occurred at intervals of 20 iterations. Therefore, it is possible to notice that the average performance increases, and the amount of learning problems decreases, as $L S_{p}$ is updated and becomes consistent with the simulated student's LS.

Following, we present results obtained through an experiment considering the initial $L S_{p}$ presented in Table 8 . In this experiment, we simulated the case in which there is no initial assumptions about student's LS. Therefore, $L S_{p}$ is initialized with cold start, which sets 0.50 to the $L S_{p}$.
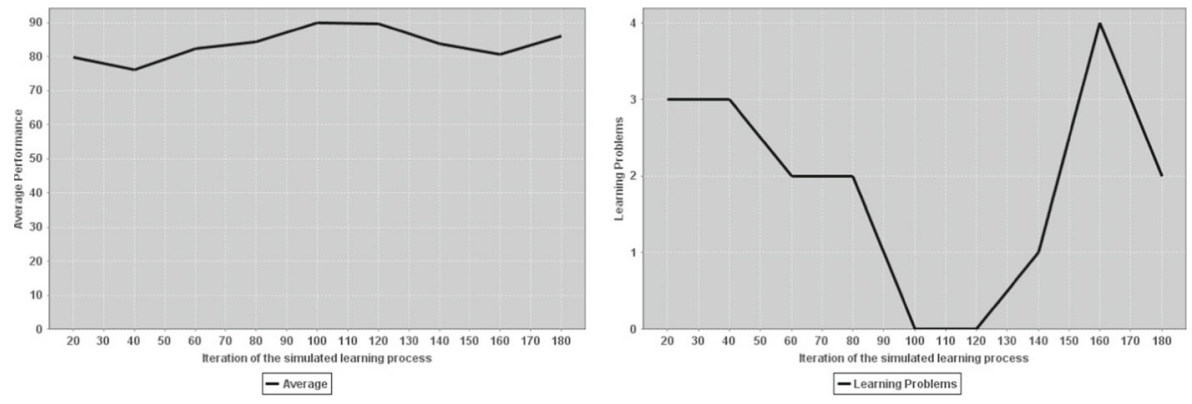

Fig. 5 Average performances and average learning problems 
Table 10 Initial $L S_{p}$ consistent with the student's actual learning style

\begin{tabular}{|c|c|c|c|c|c|c|c|}
\hline \\
\hline (A) & (R) & (S) & (I) & (Vi) & $(\mathrm{Ve})$ & $(\mathrm{Seq})$ & (G) \\
\hline 0.23 & 0.77 & 0.21 & 0.79 & 0.14 & 0.86 & 0.19 & 0.81 \\
\hline
\end{tabular}

The simulated student's real LS considered in the following experiment is \{ Reflective (strong), Intuitive (strong), Verbal (moderate), Global (balanced) \}. Figure 3 graphically presents the updating of $L S_{p}$ during the first execution of this experiment. One more time, it is important to notice that all $L S_{p}$ dimensions become consistent during the learning process, satisfying consistency and efficiency aspects.

Table 9 shows the number of iterations, the amount of learning problems (LP), and the resulting $L S_{p}$ values at the end of the simulated learning process for 10 consecutive executions of this experiment. As it can be seen, all executions produced consistent results.

Figure 4 displays performance values inferred by the simulated student at intervals of five learning sessions, obtained at the first execution of this experiment. The horizontal line represents $m$, and divides good performances from poor performances. The axis $x$ represents the iteration (learning session), and the axis $y$ shows the performance obtained by the student in the learning session. As expected, the student's performance were improved, and learning problems were reduced, as inconsistencies were eliminated from $L S_{p}$.
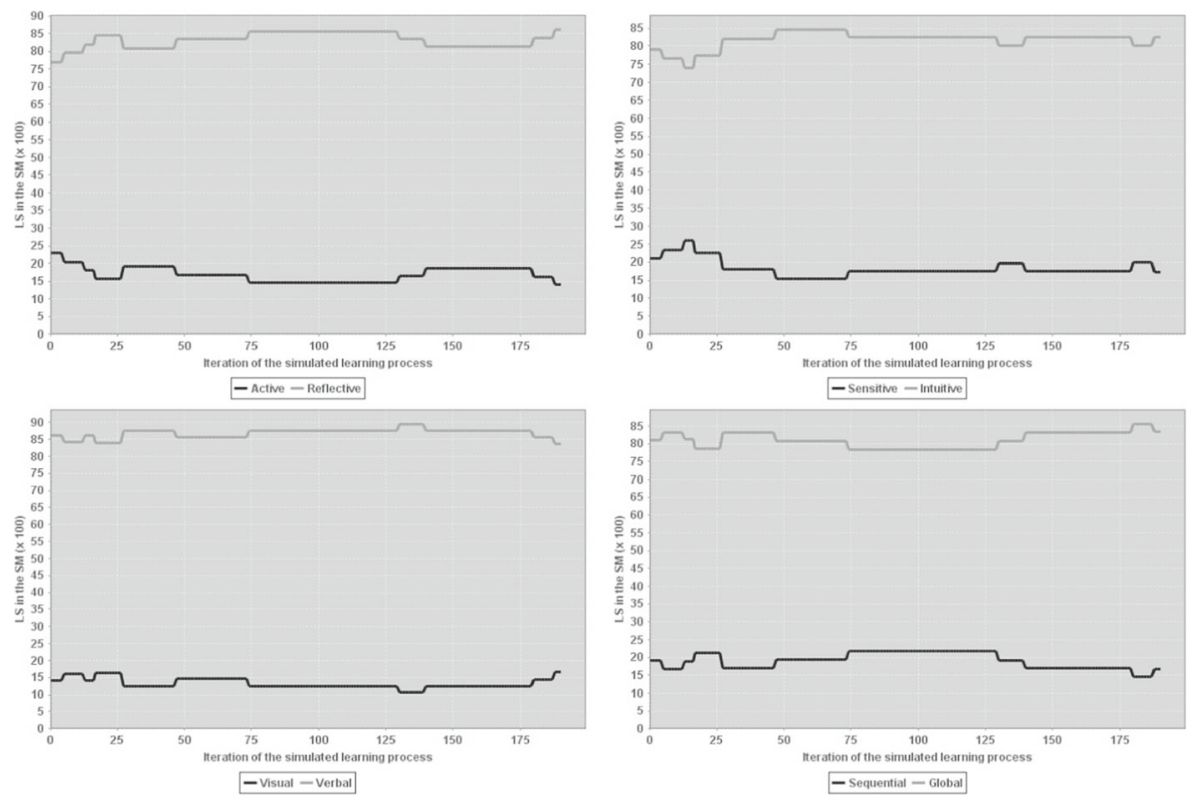

Fig. 6 Updating $L S_{p}$ throughout the simulated learning process - satisfying the maintenance aspect 


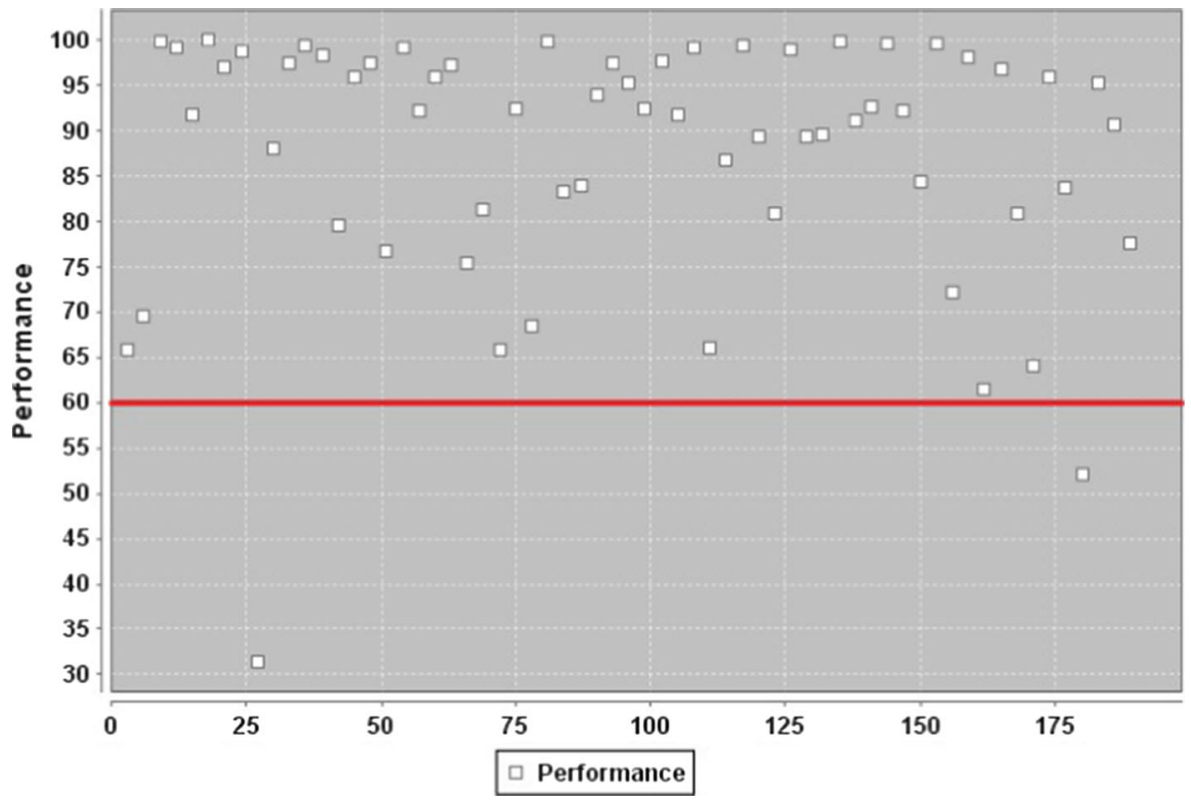

Fig. 7 Simulated student's performances - lesser number of poor performances

Figure 5 shows the average performance achieved by the simulated student, and the average learning problems in steps of 20 learning sessions. The axis $x$, in both graphs, represents the iteration (learning session). The axis $y$ show the average performance along the learning sessions, and the number of learning problems that occurred along the learning sessions (depending on the graph).

It is important to notice that, in this experiment, less iterations were necessary. This occurred because inconsistencies in the student model seem to be worse than the lack of initial information about student's LS. When the system doesn't have any initial assumption about the student's LS, it discovers them faster and provides accurate adaptivity earlier, making the learning process easier from its very beginning.

Following, it is presented results obtained through an experiment considering initial $L S_{p}$ presented in Table 10. In this experiment, the initial $L S_{p}$ were set with
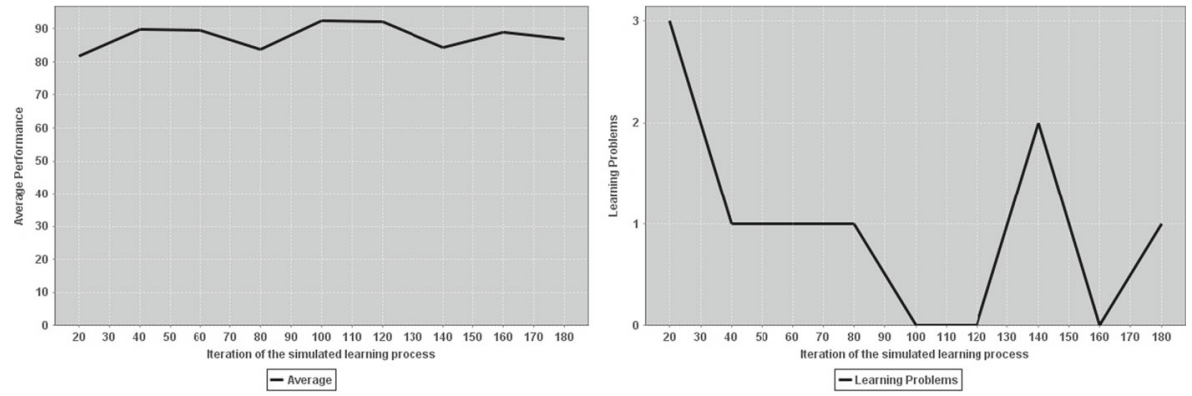

Fig. 8 Average performances and average learning problems - learning problems may occur 
results obtained from the previous experiment. Hence, the initial $L S_{p}$ are consistent with the student's actual learning style.

The student's actual learning styles considered in this experiment are equal to those considered in the previous experiment. Figure 6 graphically presents the updating of $L S_{p}$ during the execution of this experiment. It is important to notice that $L S_{p}$ remained consistent during the learning process, satisfying the maintenance aspect.

Figure 7 displays the performance values inferred by the simulated student at intervals of five learning sessions. We can notice that a lesser number of poor performances appeared in the figure.

Figure 8 shows the average performance achieved by the simulated student, and the average learning problems in steps of 20 learning sessions. It is important to observe that even when $L S_{p}$ is fully consistent with the simulated student's LS $\left(L S_{u}=0\right)$, learning problems may occur, because not only learning styles exert influence on students' performances.

It is possible to notice that in this experiment we had less learning problems and greater average performance than in previous experiments. Consequently, a lesser number of iterations was necessary in order to finish the course. Therefore, we can state that the reuse of student models in new courses is essential. The emergence of patterns could enable to share student models between different platforms. In this case, a diversity of characteristics should be shared, including LS. This practice should enable different AES to collaboratively and continuously evolve the student model, making it more consistent along the time. Next section present conclusion and future work.

\section{Conclusions and Future Work}

Modelling complex systems usually requires the use of simulation techniques, which allow us to simulate real-world systems behaviour. Stochastic simulation is frequently used to model systems whose operation cannot be captured directly by deterministic rules, as it occurred in this work.

The simulation of students has been increasingly adopted for testing AES, allowing us to observe in advance how the system behaves and the results generated. Therefore, it allows us to understand the system and its behaviour, enabling us to improve the system design.

In this context, using simulated students for testing new approaches in the field of AES is crucial, since the test and validation of these systems require considerable amounts of financial, human resources and time. Thus, it is important to evaluate the performance of a model before effectively deploying it in an educational system, due to the complexity of this task and the amount of resources required.

That said, this work presented a simulated student able to simulate performances while its learning styles preferences are matched or mismatched by the system. It is important to mention that the simulated student presented in this work takes into account some important aspects related to students performances and LS. It considers that not only LS but also many factors exert some influence on students' performances, making it harder to infer students' performances based only on fixed 
behavioural pattern rules, because students' behaviour and performance may be influenced by other factors besides LS. Some of these factors are pointed out by Haider et al. (2010), Graf et al. (2008), Kinshuk and Graf (2009), Alfonseca et al. (2006), Graf et al. (2009), and Messick (1976).

In this way, the simulated student presented in this work has been developed based on recent investigations on how LS may exert influence on students' performances, as stated by Felder and Silverman (1988), Haider et al. (2010), Graf et al. (2008), Kinshuk and Graf (2009), Alfonseca et al. (2006), and Graf et al. (2009). This issue have been largely studied, and many knowledge may be extracted from the literature, enabling researchers to automate tests through simulated students. It means that it is possible to find consistent information on how student learning styles guides behaviour and affects performance. Consequently, it is possible to create a mathematical model that represents this knowledge, implement and execute it using computers.

It is important to notice that there are uncertainty aspects on how learning styles exert influence on students' performances, and it definitely is not a deterministic process, as previously stated by (Felder and Silverman 1988). But, it is known that when the learning environment, its features, its content, its navigation model and its resources are adapted to a particular student's learning styles, chances of getting good performances increases. This decreasing of difficulty and consequent improvement of performance is stochastically modelled by the simulated student presented in this paper.

The simulated student module allowed us to test, adjust and improve our approach since the very beginning, optimizing the development process. Different strategies for updating the student model were analysed, and their efficiency and effectiveness were compared. The best strategy has been found, proving the hypotheses 1 and 2 presented in introduction. A deep study on the chosen strategy was possible by the use of simulation, proving the hypothesis 3. This finding was possibility by the use of simulation. Without this possibility, the comparison between different strategies, and the carefull observation of their behaviours would be impossible, due to reasons exposed before in this work.

The strategies were analysed considering three important aspects: consistency, efficiency and maintenance. The use of simulation was critical at this point, allowing detailed comparison of the strategies. Therefore, it was possible to quickly discard less efficient strategies, focusing efforts on the most promising strategy. Without the use of simulation, the clear observation of the efficiency of each strategy and the discover of the most promising would be impossible. This is because this observation requires a process with considerable number of iterations, so you can be confident and clear about what is the most efficient. In this case, the comparison of strategies by conducting experiments with human subjects would require extremely long time, certainly impeding this work and its promising results.

As a result, the best strategy have been chosen, and it is currently being deployed in Moodle (Moodle 2010). Therefore, on a future work, we will test it with real students, and compare the results with those presented in this paper. In this opportunity, we will be interested in observe if the average performance of real students increase as their $L S_{p}$ converges to their real learning styles, as it happened with simulated students. 
Acknowledgments I acknowledge FACOM/UFU (Faculty of Computing) and PROPP/UFU (Pro-Dean for Research and Graduate Studies) at Federal University of Uberlandia/Brazil for all the support during the execution of this work.

\section{References}

Abdullah, S., \& Cooley, R. (2002). Using simulated students to evaluate an adaptive testing system. In Proceedings of international conference on computers in education, 2002 (pp. 614-618). IEEE.

Al-Dujaily, A., \& Ryu, H. (2006). A relationship between e-learning performance and personality. In Sixth international conference on advanced learning technologies, 2006 (pp. 84-86). IEEE.

Alfonseca, E., Carro, R., Martín, E., Ortigosa, A., Paredes, P. (2006). The impact of learning styles on student grouping for collaborative learning: a case study. User Modeling and User-Adapted Interaction, 16(3), 377-401.

Bajraktarevic, N., Hall, W., Fullick, P. (2003). Incorporating learning styles in hypermedia environment: Empirical evaluation. In Proceedings of the workshop on adaptive hypermedia and adaptive webbased systems (pp. 41-52).

Bostrom, L. (2011). Students' learning styles compared with their teachers' learning styles in secondary schools. Institute for Learning Styles Journal, 1, 17-38.

Bravo, J., \& Ortigosa, A. (2006). Validating the evaluation of adaptive systems by user profile simulation. In Proceedings of workshop held at the 4th international conference on adaptive hypermedia and adaptive web-based systems (AH2006) (pp. 479-483).

Brusilovsky, P. (2001). Adaptive educational hypermedia. In International PEG conference (pp. 8-12). Citeseer.

Coffield, F., Moseley, D., Hall, E., Ecclestone, K. (2009). Learning styles and pedagogy in post-16 learning: a systematic and critical review. National Centre for Vocational Education Research (NCVER).

Entwistle, N. (1981). Styles of learning and teaching. Wiley Chichester.

Felder, R., \& Silverman, L. (1988). Learning and teaching styles in engineering education. Journal of Engineering Education, 78(7), 674-681.

Friedel, C., \& Rudd, R. (2006). Creative thinking and learning styles in undergraduate agriculture students. Journal of Agricultural Education, 47(4), 102.

Geiss, C. (2009). Stochastic Modeling. users.jyu.fi/ geiss/scripts/stochastic-models.pdf, 1.

Goguadze, G., Sosnovsky, S., Isotani, S., McLaren, B. (2011). Evaluating a bayesian student model of decimal misconceptions. In Proceedings of the 4th international conference on educational data mining.

Goldberg, D. (1989). Genetic algorithms in search, optimization, and machine learning. Addison-wesley.

Graf, S., \& Kinshuk. C. (2009). Advanced adaptivity in learning management systems by considering learning styles. In Proceedings of the 2009 IEEE/WIC/ACM international joint conference on web intelligence and intelligent agent technology (Vol. 03, pp 235-238). IEEE Computer Society.

Graf, S., \& Kinshuk, C. (2010). A flexible mechanism for providing adaptivity based on learning styles in learning management systems. In 10th IEEE international conference on advanced learning technologies (pp. 30-34). IEEE.

Graf, S., \& Kinshuk, K. (2007). Providing adaptive courses in learning management systems with respect to learning styles. In Proceedings of world conference on E-learning in corporate, government, healthcare, and higher education 2007 (pp. 2576-2583).

Graf, S., Lan, C., Liu, T., et al. (2009). Investigations about the effects and effectiveness of adaptivity for students with different learning styles. In 2009 9th IEEE international conference on advanced learning technologies (pp. 415-419). IEEE. 
Graf, S., \& Lin, T. (2007). Analysing the relationship between learning styles and cognitive traits. In Seventh IEEE international conference on advanced learning technologies, 2007. ICALT 2007 (pp. 235-239). IEEE.

Graf, S., \& Liu, T. (2008). Identifying learning styles in learning management systems by using indications from students' behaviour. In Eighth IEEE international conference on advanced learning technologies, 2008. ICALT'08 (pp. 482-486). IEEE.

Graf, S., Liu, T.-C., Kinshuk, C. (2008). Interactions between students learning styles, achievement and behaviour in mismatched courses. In Proceedings of the international conference on cognition and exploratory learning in digital age (CELDA 2008) (pp. 223-230). IADIS International Conference.

Haider, M., Sinha, A., Chaudhary, B. (2010). An investigation of relationship between learning styles and performance of learners. International Journal of Engineering Science and Technology, 2(7), 2813-2819.

Honey, P., \& Mumford, A. (1992). The manual of learning styles, 3Rev Edn. Peter Honey Publications.

IEEE (2010). LOM (Learning Object Metadata). IEEE Learning Technology Standards Committee, http://ltsc.ieee.org/wg12/index.html.

Kinshuk, L.iu., T., \& Graf, S. (2009). Coping with Mismatched Courses: Students' behaviour and performance in courses mismatched to their learning styles. Educational Technology Research and Development, 57(6), 739-752.

Kolb, D., et al. (1984). Experiential learning: experience as the source of learning and development. Prentice-Hall Englewood Cliffs.

Kuljis, J., \& Liu, F. (2005). A comparison of learning style theories on the suitability for elearning. In Proceedings of the IASTED conference on web technologies, applications, and services (pp. 191197).

Lim, H., Lee, S., Nam, K. (2007). Validating e-learning factors affecting training effectiveness. International Journal of Information Management, 27(1), 22-35.

Mertz, J. (1997). Using a simulated student for instructional design. International Journal of Artificial Intelligence in Education (IJAIED), 8, 116-141.

Messick, S. (1976). Personal styles and educational options. Individuality in Learning, 327-368.

Meyn, S., Tweedie, R., Glynn, P. (1996). Markov chains and stochastic stability. Springer London.

Moodle (2010). http://www.moodle.org/.

Mosakhani, M., \& Jamporazmey, M. (2010). Introduce critical success factors (CSFs) of elearning for evaluating e-learning implementation success. In 2010 international conference on educational and information technology (ICEIT) (vol. 1, pp. 51-224). IEEE.

Myers, I., McCaulley, M., Most, R. (1985). Manual: a guide to the development and use of the MyersBriggs Type Indicator. Consulting Psychologists Press Palo Alto.

Pask, G. (1976). Styles and strategies of learning. British journal of educational psychology.

Prodan, A., \& Prodan, R. (2001). Stochastic simulation and modelling. ACM Journal of Education Resources in Computing, 1, 461-466.

Sangineto, E., Capuano, N., Gaeta, M., Micarelli, A. (2008). Adaptive course generation through learning styles representation. Universal Access in the Information Society, 7(1), 1-23.

Santos, O., \& Boticario, J. (2008). Recommendation strategies for promoting eLearning performance factors for all. In 6th workshop on intelligent techniques for web personalization \& recommender systems in conjunction with The 23nd AAAI conference on artificial intelligence-2008.

Sutton, R., \& Barto, A. (1998). Reinforcement learning: an introduction, (vol. 116). Cambridge Univ Press.

Terry, R.E., Harb, J.N., Hurt, P., Williamson, K. (1995). Teaching through the cycle: application of learning style theory to engineering education at Brigham Young University. Brigham Young University Press.

Vanlehn, K., Ohlsson, S., Nason, R. (1994). Applications of simulated students: an exploration. Journal of artificial intelligence in education, 5, 135-135.

Vasilyeva, E., Pechenizkiy, M., Puuronen, S. (2006). The challenge of feedback personalization to learning styles in a web-based learning system. In Sixth international conference on advanced learning technologies, 2006, (pp. 1143-1144). IEEE.

Virvou, M., Manos, K., Katsionis, G. (2003). An evaluation agent that simulates students' behaviour in intelligent tutoring systems. In IEEE international conference on systems, man and cybernetics, 2003 (vol. 5, pp. 4872-4877). IEEE. 
Vizcaino, A., \& Du Boulay, B. (2002). Using a simulated student to repair difficulties in collaborative learning. In ICCE' 02 proceedings of the international conference on computers in education. Washington, DC: ACM, IEEE Computer Society.

Wojtusiak, J., Warden, T., Herzog, O. (2012). Machine learning in agent-based stochastic simulation: Inferential theory and evaluation in transportation logistics. Computers \& Mathematics with Applications. doi:10.1016/j.camwa.2012.01.079.

Zhang, L., Zhang, X., Duan, Y., Fu, Z., Wang, Y. (2010). Evaluation of learning performance of E-learning in China: a methodology based on change of internal mental model of learners. Turkish Online Journal of Educational Technology-TOJET, 9(1), 13. 